\title{
ISPRS Workshop on Semantic Scene Analysis and 3D Reconstruction from Images and Image Sequences (Semantics3D)
}

\author{
Part of the ISPRS Geospatial Week 2019
}

\author{
Enschede, The Netherlands - June 10 - 11, 2019
}

Automated 3D reconstruction and extraction of semantic information from images and image sequences are important topics of research in Photogrammetry, Remote Sensing, GIS, and Computer Vision. Semantics3D was introduced as a workshop following up a series of earlier ISPRS events related to photogrammetric 3D reconstruction (PhotoGA), automatic object detection for city models, road databases and traffic analysis (CMRT), and image sequence analysis (ISA). The workshop addressed researchers and practitioners from universities, research institutes, industry, government organizations, and private companies. The range of topics covered by the workshop was reflected by the terms of reference of the cooperating ISPRS working groups:

- WG II/2 Point Cloud Generation

- WGII/4 3D Scene Reconstruction and Analysis

- WGII/5 Dynamic Scene Analysis

- WGII/6 Large-scale Machine Learning for Geospatial Data Analysis

- $\quad$ ICWG II/III Pattern Recognition in Remote Sensing

Prospective authors were invited to submit full papers of a maximum length of 8 pages. In addition, authors of application-oriented work could submit extended abstracts. The organizing committee has received 19 full papers for review. The submitted full papers were subjected to a rigorous double blind peer review process. Based on the reviews, 10 papers by authors coming from six countries were accepted. This number corresponds to an acceptance rate of 53\%. Each full paper was reviewed by a minimum of two members of the scientific committee, while most of them by three.

In addition, we received 17 extended abstracts for review. The abstracts were also reviewed by at least two members of the scientific committee, and the authors of 14 abstracts were invited to submit full papers to the workshop. We received 13 full papers based on these selected abstracts. Along with eight papers that did not pass the full paper peer review, they were published in the International Archives of the Photogrammetry, Remote Sensing and Spatial Information Science.

All accepted full papers and nine selected papers that were accepted for the Archives were presented orally at the workshop consisting of seven oral sessions. Two of these sessions were shared with the UAV-g and Indoor3D workshops, respectively. The remaining twelve papers were presented during two joint poster sessions with other GSW 2019 workshops. In addition, the programme of the Semantics3D workshop featured keynotes by Prof. Margarita Chli from ETH Zurich (Switzerland), shared with UAV-g, and Prof. Avideh Zakhor from the University of California (USA), in a shared session with the Indoor3D workshop.

The Program Committee consisted of the following persons:

- Ahmed Alamouri, Technische Universität Braunschweig, Germany

- Claus Brenner, Leibniz Universtität Hannover, Germany

- Ian Cherabier, ETH Zurich, Switzerland

- Andrea Fusiello, University of Udine, Italy

- Markus Gerke, Technische Universität Braunschweig, Germany

- Norbert Haala, University of Stuttgart, Germany

- Petra Helmholz, Curtin University, Australia

- Ludwig Hoegner, Technische Universitaet Muenchen, Germany

- Siavash Hosseinyalamdary, University of Twente, The Netherlands

- Mehdi Maboudi, Technische Universität Braunschweig, Germany

- Clément Mallet, Institut Géographique National (IGN), France

- Eleonora Masset, University of Udine, Italy

- Martin Oswald, ETH Zurich, Switzerland

- José Pena, Institute of Agricultural Sciences, CSIC, Spain

- Rongjun Qin, The Ohio State University, USA

- Camillo Ressl, Vienna University of Technology, Austria

- $\quad$ Audrey Richard, ETH Zurich, Switzerland

- Ribana Roscher, University of Bonn, Germany

- Mathias Rothermel, ETH Zurich, Switzerland 
- Franz Rottensteiner, Leibniz Universität Hannover, Germany

- Vasit Sagan, St. Louis University, USA

- Konrad Schindler, ETH Zurich, Switzerland

- Mozdeh Shabasi, University of Calgary, Canada

- Jie Shan, Purdue University, USA

- Uwe Stilla, Technische Universitaet Muenchen, Germany

- Devis Tuia, Wageningen University, The Netherlands

- Bruno Vallet, Institut Géographique National (IGN), France

- Yury Vizilter, State Research Institute of Aviation Systems, Russia

- $\quad$ Michele Volpi, ETH Zurich, Switzerland

- $\quad$ Ruisheng Wang, University of Calgary, Canada

- Jan Dirk Wegner, ETH Zurich, Switzerland

- Martin Weinmann, Karlsruhe Institute of Technology, Germany

- $\quad$ Bo Wu, The Hong Kong Polytechnic University, Hong Kong

- $\quad$ Alper Yilmaz, The Ohio State University, USA

The editors wish to thank all contributing authors, the members of the Program Committee, and Francesco Nex for maintaining the workshop web site. In addition, we like to express our thanks to the ISPRS Geospatial Week without whom this event could not have taken place.

Hannover and Columbus, June 10, 2019

Franz Rottensteiner and Alper Yilmaz 